\title{
NUMERICAL SIMULATION OF TURBULENT TRANSITIONAL FLOW AROUND AN ELASTICALLY SUPPORTED AEROFOIL
}

\author{
PETR SvÁČEK \\ Czech Technical University in Prague, Faculty of Mechanical Engineering, \\ Dep. of Technical Mathematics, Karlovo nám. 13, 12135 Praha 2, Czech Republic \\ petr.svacek@fs.cvut.cz
}

[Received: March 31, 2016, Accepted: July 12, 2016]

\begin{abstract}
In this paper the numerical simulation of the interaction of fluid flow with a flexibly supported aerofoil is addressed. Particularly, the turbulent flow model with a laminar-turbulence transition is considered. The transitional model is based on the two equation $k-\omega$ turbulence model, where the additional two equations for the intermittency and transitional onset Reynolds number are included. The motion of the computational domain is treated with the aid of the arbitrary Lagrangian-Eulerian method. The attention is paid mainly to the numerical approximation of the complex nonlinear coupled problem. The numerical results are shown for aeroelastic response of the aerofoil.
\end{abstract}

Mathematical Subject Classification:

Keywords: turbulence models, transition, finite element method, aeroelasticity

\section{INTRODUCTION}

Numerical approximation of fluid-structure interaction (FSI) problems has recently become important in many technical applications in turbomachinery, aerospace engineering, biomechanics. In technical practice particularly the prediction of aeroelastic instability as flutter is important [2]. The aeroelastic instability region is usually treated with the aid of a linearized approach [1. Such an approach can provide necessary conditions to guarantee safety, but the transient growth induced by external excitation can lead to structural failure even though the system is aeroelastically stable, see [3]. The problem of transient aeroelastic response was addressed in [4, where the combined aeroelastic behaviour and gust response of a flexible aerofoil was explored theoretically. In [5] the FSI problem of gust response of a flexible typical section was investigated in terms of both high- and low-fidelity simulations. The use of accurate aeroelastic simulations is particularly attractive because it reduces the development risk, the number of experiments and the possible design modifications. However, computational efforts associated with high fidelity aeroelastic models currently precludes their direct use in industry [6]. Acceleration of time-accurate high fidelity aeroelastic simulation algorithms has therefore become an active area of research. The most common solution strategy is the so-called partitioned approach, 
which de-couples the original FSI problem and uses specialized solvers for each subproblem (see [7]). The coupling is then enforced at the fluid-solid interface by suitable interface conditions.

The other thing is that for approximation of FSI problem the applied numerical method should be able to treat the moving domain/meshes. The most popular method is the Arbitrary Lagrangian-Eulerian (ALE) method, see [8]. There are several possibilities how to apply the finite element method (FEM) [9]. Usually in order to provide higher order accuracy or stability some additional assumptions on the ALE are required, see [10]. Particularly the so-called geometrical conservation law is important [1].

Moreover, in order to apply the FEM for numerical simulation of incompressible flow problem must overcome several sources of instability. One instability is caused by the incompatibility of the pressure and velocity pairs of finite elements, cf. [12]. The other instability is due to the dominating convection terms, see 13. There is another less well-studied instability source in the Galerkin discretization method related to a possible poor resolution of pressure, see [14, 15], 16].

The numerical simulation of interactions of flow with vibrating aerofoil was considered in [17. Nevertheless the FSI problems usually include high Reynolds number flows, where the turbulence effects need to be included [18. In [19] the interaction of a three degrees of freedom (DOF) airfoil was addressed with the turbulent flow modeled by the Reynolds averaged Navier-Stokes (RANS) equations combined with Spalart-Allmaras or $k-\omega$ turbulence models. The flow was also modelled by the RANS equations combined with the $k-\omega$ turbulence model in the paper [20, where the developed in-house FEM code was applied to the numerical simulation of the aeroelastic interaction of flexibly supported 2-DOF aerofoil with the 2D incompressible viscous turbulent flow subjected to a sudden gust. The developed method was successfully tested comparing the results with the study published in [4, [21], where the aerofoil response to the gust was computed by a commercial CFD program code.

The same FSI problem was studied in [22], where the aeroelastic aerofoil response to the gust computed for the turbulent airflow was compared with the laminar flow. In this case, the appearance of the flow separation can significantly influence the aeroelastic response. The application of the turbulent model on the other hand consider the boundary layer to be turbulent on the whole surface of the aerofoil. In reality the transition from laminar to turbulent flow exists on the surface of the aerofoil. The transition in technical applications can be modeled with the aid of different approaches, see [23], 24]. One class of these models is based on the use of an equation for the intermittency coefficient [25]. For this equation usually empirical correlations need to be involved with application of some non-local operations (as determination of the boundary layer thickness). The use of these operations is usually computationally expensive on non-structured grids. In order to get rid of these non-local operations another approach was proposed in [26] and also [27. Here, the intermittency equation is coupled to the additional modeled variable and onset criteria are then related to this new local variable. 
Although there are numerous publications devoted to the transitional model, there are not as many papers interested in the numerical simulation of FSI problems with transitional model included. In 28 the unsteady transitional flow over an oscillating NACA 0012 aerofoil was numerically investigated, showing that the transition from laminar to turbulent state and relaminarization occur widely in time and in space. In [29] the 2 DOF bending/torsion flutter characteristics of the NLR 7301 section was numerically investigated and the natural transition was modelled. In 30 low amplitude self-sustained pitch oscillations in the transitional Reynolds number regime are studied numerically showing the importance of the laminar separation of the boundary layer near the trailing edge for initiating and sustaining the pitching oscillations. In 31] the effect of freestream turbulence on small-amplitude limit-cycle oscillations of an aerofoil is investigated with the aid of a correlation-based transitional model.

In the present paper Menter's transitional turbulence model is described and applied for numerical simulation of $2 \mathrm{D}$ viscous incompressible flow past a flexibly supported aerofoil. First, the mathematical model of the considered problem is described and its numerical approximation is shortly explained. Further, the applicability of the method is shown on a benchmark problem.

\section{Mathematical Description}

2.1. Flow model. The mathematical formulation of the problem consists of the flow model, the structure model and the interface conditions. We consider the twodimensional time dependent computational domain $\Omega_{t} \subset \mathbb{R}^{2}$ with the Lipschitz continuous boundary $\partial \Omega_{t}$, see Figure 11. The fluid motion in the domain $\Omega_{t}$ is modeled using the Reynolds averaged Navier-Stokes system of equations in $\Omega_{t}$

$$
\frac{\partial u_{i}}{\partial t}+\frac{\partial\left(u_{i} u_{j}\right)}{\partial x_{j}}-\frac{\partial}{\partial x_{j}}\left(-p \delta_{i j}+2 \nu_{\text {eff }} S_{i j}\right)=0, \quad \frac{\partial u_{i}}{\partial x_{i}}=0,
$$

where $\boldsymbol{u}=\left(u_{1}, u_{2}\right)$ is the mean part of the fluid velocity vector, $S_{i j}=\frac{1}{2}\left(\frac{\partial u_{i}}{\partial x_{j}}+\frac{\partial u_{j}}{\partial x_{i}}\right)$ are the components of $\boldsymbol{S}=\boldsymbol{S}(\boldsymbol{u})$ the symmetric part of the gradient of $\boldsymbol{u}, p$ is the mean part of the kinematic pressure (i.e., the pressure divided by the constant fluid density $\rho), \nu_{\text {eff }}=\nu+\nu_{T}, \nu$ is the kinematic viscosity of the fluid (i.e. the viscosity divided by the density $\rho), \nu_{T}$ is a turbulent viscosity (obtained by an additional model), see [18, 32].

The system (2.1) is equipped with an initial condition $\boldsymbol{u}(x, 0)=\boldsymbol{u}_{0}(x)$ for $x \in \Omega_{0}$ and with boundary conditions prescribed on the mutually disjoint parts $\Gamma_{D}, \Gamma_{O}$ and $\Gamma_{W t}$ of the boundary $\partial \Omega=\Gamma_{D} \cup \Gamma_{O} \cup \Gamma_{W t}$ :
a) $\boldsymbol{u}=\boldsymbol{u}_{D} \quad$ on $\Gamma_{D}$,
b) $\boldsymbol{u}=\boldsymbol{w}_{D} \quad$ on $\Gamma_{W t}$,
c) $-2 \nu_{\text {eff }} S_{i j} n_{j}+p n_{i}=0$
on $\Gamma_{O}$,

where $\boldsymbol{w}_{D}$ is the velocity of the boundary $\Gamma_{W t}$ and $\boldsymbol{u}_{D}$ is the prescribed inlet velocity in the form $\boldsymbol{u}_{D}=\left(U_{\infty}, V_{g}(t)\right)^{T}$. Here $U_{\infty}$ is the far field velocity and $V_{g}(t)$ is the vertical gust velocity. 


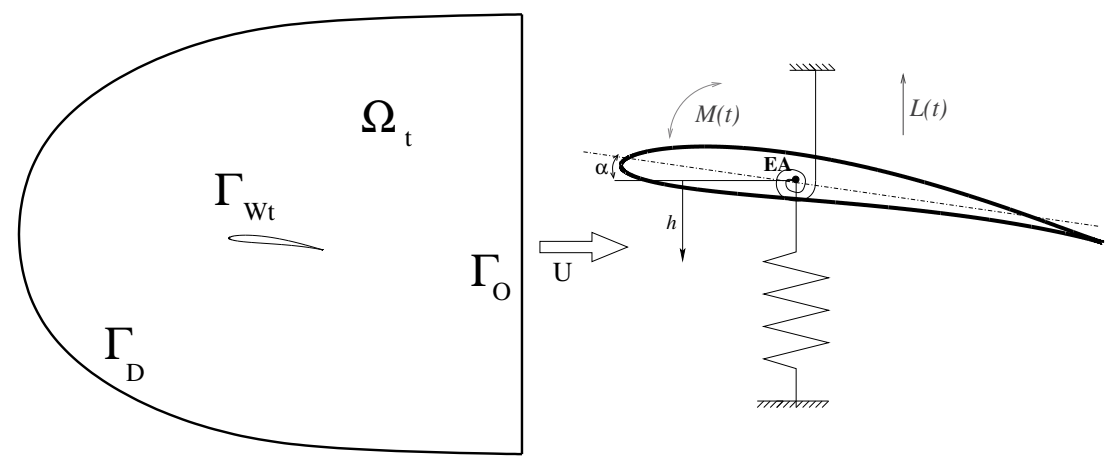

Figure 1. Sketch of the computational domain $\Omega_{t}$ its boundary $\partial \Omega_{t}$ (left) and the flexibly supported aerofoil model (right)

2.2. SST turbulence model. In order to enclose the system 2.1 the turbulent viscosity is modeled with the aid of SST $k-\omega$ turbulence model, see 33. The transition is treated with the aid of the $\gamma-\overline{R e}_{\theta t}$ model is used, see [26]. The SST turbulence model is a modification of the $k-\omega$ turbulence model for which the turbulent viscosity is given by $\nu_{T}=\frac{k}{\omega}$ Here, the turbulent kinetic energy $k=k(x, t)$ and the turbulent specific dissipation rate $\omega=\omega(x, t)$ are modeled by

$$
\begin{aligned}
\frac{\partial k}{\partial t}+\frac{\partial\left(u_{i} k\right)}{\partial x_{i}} & =\gamma_{\mathrm{eff}} P_{k}-\beta^{*} \omega k \overline{\gamma_{\mathrm{eff}}}+\frac{\partial}{\partial x_{i}}\left(\varepsilon_{k} \frac{\partial k}{\partial x_{i}}\right), \\
\frac{\partial \omega}{\partial t}+\frac{\partial\left(u_{i} \omega\right)}{\partial x_{i}} & =P_{\omega}-\beta \omega^{2}+\frac{\partial}{\partial x_{i}}\left(\varepsilon_{\omega} \frac{\partial \omega}{\partial x_{i}}\right)+C_{D},
\end{aligned}
$$

where the production and the destruction terms are modified due in the first equation using the effective intermittency coefficient $\gamma_{\text {eff }}$ and $\bar{\gamma}_{\text {eff }}=\max \left(\min \left(\gamma_{\text {eff }}, 1\right), 0.1\right)$. Further, the viscosity coefficients are given by $\varepsilon_{k}=\nu+\sigma_{k} \nu_{T}$ and $\varepsilon_{\omega}=\nu+\sigma_{\omega} \nu_{T}$ and the source terms $P_{k}$ and $C_{D}$ are defined by

$$
P_{k}=\nu_{T} S_{i j} S_{i j}, \quad C_{D}=\frac{\sigma_{D}}{\omega}\left(\frac{\partial k}{\partial x_{i}} \frac{\partial \omega}{\partial x_{i}}\right)^{+},
$$

and $P_{\omega}=\frac{\alpha_{\omega} \omega}{k} P_{k}$. The closure coefficients $\beta, \beta^{*}, \sigma_{k}, \sigma_{\omega}, \alpha_{\omega}, \sigma_{D}$ are chosen from [33, see also [34] or [18. Equations 2.3 are equipped with boundary conditions
a) $\quad k=k_{\infty}, \quad \omega=\omega_{\infty} \quad$ on $\Gamma_{D}$,
b) $\quad k=0, \quad \omega=\omega_{\text {wall }} \quad$ on $\Gamma_{W t}$,
c) $\quad \frac{\partial k}{\partial \boldsymbol{n}}=0, \quad \frac{\partial \omega}{\partial \boldsymbol{n}}=0, \quad$ on $\Gamma_{O}$.

2.3. Transitional model. The transition from laminar to turbulence regimes is modeled with the aid of Menter's $\gamma-\overline{R e}_{\theta t}$ model, see [26], where the equation for the 
intermittency coefficient $\gamma$ reads

$$
\frac{\partial \gamma}{\partial t}+\frac{\partial\left(u_{i} \gamma\right)}{\partial x_{i}}=P_{\gamma}-E_{\gamma}+\frac{\partial}{\partial x_{i}}\left(\left(\nu+\frac{\nu_{T}}{\sigma_{f}}\right) \frac{\partial \gamma}{\partial x_{i}}\right),
$$

where $P_{\gamma}=P_{\gamma, 1}-c_{e 1} \gamma P_{\gamma, 1}$ and $E_{\gamma}=c_{e 2} \gamma E_{\gamma, 1}-E_{\gamma, 1}$ are the transition source and destruction terms, respectively. Here, we set $P_{\gamma, 1}=F_{\text {length }} c_{a 1} S \sqrt{\gamma F_{\text {onset }}}, E_{\gamma, 1}=$ $c_{a 2} \Omega \gamma F_{\text {turb }}$. Further, $S$ and $\Omega$ are the strain rate and vorticity magnitudes and the transition onset is modeled by $F_{\text {onset }}=\left(F_{\text {onset2 }}-F_{\text {onset3 }}\right)^{+}$, where $F_{\text {onset2 }}=$ $\min \left(\max \left(F_{\text {onset } 1}, F_{\text {onset } 1}^{4}\right), 2\right), F_{\text {onset } 1}=\frac{R e_{V}}{2.193 R e_{\theta c}}, R e_{V}=\frac{y^{2} S}{\nu}$. Further we set $F_{\text {onset3 }}=$ $\max \left(1-\left(\frac{R_{T}}{2.5}\right)^{3}, 0\right), R_{T}=\frac{k}{\nu \omega}$, and $F_{\text {turb }}=e^{-\left(R_{T} / 4\right)^{4}}$, where $y$ denotes the wall distance and $R e_{\theta t}$ is the transition Reynolds number. The following constants for the intermittency equation were used $c_{e 1}=1, c_{a 1}=2, c_{e 2}=50, c_{a 2}=0.06, \sigma_{f}=1$. Further, $R e_{\theta c}$ is the critical Reynolds number given by an empirical correlation, and another empirical correlation is used for the function $F_{\text {length }}$, which controls the length of the transition region. The correlations are based on newly defined transported unknown $\overline{R e}_{\theta t}$ governed by the ALE form of the equation

$$
\frac{\partial \overline{R e}_{\theta t}}{\partial t}+\frac{\partial\left(u_{i} \overline{R e}_{\theta t}\right)}{\partial x_{i}}=P_{\theta t}+\frac{\partial}{\partial x_{i}}\left(\sigma_{\theta t} \nu_{\text {eff }} \frac{\partial \overline{R e}_{\theta t}}{\partial x_{i}}\right),
$$

where the source term $P_{\theta t}$ is given by

$$
P_{\theta t}=c_{\theta t} \frac{\rho}{t_{\infty}}\left(R e_{\theta t}-\overline{R e}_{\theta t}\right)\left(1-F_{\theta t}\right),
$$

$c_{\theta t}=0.03, \sigma_{\theta t}=2, t_{\infty}=500 \nu / U^{2}$ is the time scale, $U$ is the local magnitude of the velocity $U=\|\boldsymbol{u}\|_{2}$ and the blending function $F_{\theta t}$ is defined as

$$
\begin{gathered}
F_{\theta t}=\min \left(1, \max \left(F_{\text {wake }} e^{-(y / \delta)^{4}}, 1-\left(\frac{\gamma-1 / c_{e 2}}{1-1 / c_{e 2}}\right)\right)\right), \\
\delta=\frac{375 \Omega y}{U} \theta, \theta=\frac{\overline{R e}_{\theta t} \nu}{U}, F_{\text {wake }}=e^{-\left(R e_{\omega} / 10^{5}\right)^{2}},
\end{gathered}
$$

and $R e_{\omega}=\frac{\omega y^{2}}{\nu}$. The source term $P_{\theta t}$ on the right hand side of equation 2.6 includes also the Reynolds number $R e_{\theta t}$ given by an empirical correlations.

The transitional model $(2.5)$ and $(2.6)$ is equipped with the boundary conditions
a) $\quad \gamma=1, \quad \overline{R e}_{\theta t}=\overline{R e}_{\theta t \infty}$
on $\Gamma_{D}$,
b) $\quad \frac{\partial \gamma}{\partial \boldsymbol{n}}=0, \quad \frac{\partial \overline{R e}_{\theta t}}{\partial \boldsymbol{n}}=0$
on $\Gamma_{W t} \cup \Gamma_{O}$

2.4. Empirical correlations. In order to enclose the model, the empirical correlations published in 35 are used. First, the length of the transition is controlled by $F_{\text {length }}=F_{\text {length }}\left(\overline{R e}_{\theta t}\right)$. Further, the transitional onset momentum thickness Reynolds number $R e_{\theta t}$ is correlated to pressure gradient $\lambda_{\theta}$ and to turbulence intensity $T u$. Further, the correlation for $R e_{\theta t}$ and for the critical Reynolds number are specified, see [35] or [24]. 
2.5. ALE formulation. In order to practically treat the motion of the domain $\Omega_{t}$, the Arbitrary Lagrangian-Eulerian (ALE) method is used, see [8]. The ALE mapping $\mathcal{A}: \Omega_{0}^{\text {ref }} \mapsto \Omega_{t}, \mathcal{A}=\mathcal{A}(\xi, t)=\mathcal{A}_{t}(\xi)$ defined for all $t \in(0, T)$ and $\xi \in \Omega_{0}^{\text {ref }}=\Omega_{0}$ is assumed to be smooth and to have smooth bounded Jacobian $\mathcal{J}(x, t)$ Furthermore, by $D^{\mathcal{A}} / D t$ the ALE derivative is denoted (i.e. the derivative with respect to the reference configuration) and $\boldsymbol{w}_{D}$ denotes the domain velocity (i.e. velocity of the point $x=\mathcal{A}(\xi, t)$ with a given reference $\xi \in \Omega_{0}^{r e f}$. The ALE derivative is then related to the time derivative by (see also [9])

$$
\frac{D^{\mathcal{A}} f}{D t}(x, t)=\frac{\partial f}{\partial t}(x, t)+\boldsymbol{w}_{D}(x, t) \cdot \nabla f(x, t) .
$$

In order to approximate the equations on time dependent domains, the time derivative in equations $(2.1), \sqrt{2.3}, \sqrt{2.5}$ and $(2.6)$ are replaced by the ALE time derivative using the formula 2.7), which also modifies the convection term. In the practical computation this is not complicated and thus for the sake of brevity this description is left out.

2.6. Structure model. The flow model is coupled with the structure model representing the flexibly supported aerofoil (see Figure 1). The aerofoil can be vertically displaced by $h$ (downwards positive) and rotated by angle $\alpha$ (clockwise positive). The nonlinear equations of motion then read (see [9])

$$
\begin{array}{r}
m \ddot{h}+S_{\alpha} \ddot{\alpha} \cos \alpha-S_{\alpha} \dot{\alpha}^{2} \sin \alpha+k_{h} h=-L(t), \\
S_{\alpha} \ddot{h} \cos \alpha+I_{\alpha} \ddot{\alpha}+k_{\alpha} \alpha=M(t) .
\end{array}
$$

where $m$ is the mass of the aerofoil, $S_{\alpha}$ is the static moment around the elastic axis (EA), and $I_{\alpha}$ is the inertia moment around EA. The parameters $k_{h}$ and $k_{\alpha}$ denote the stiffness coefficients. On the right-hand side the aerodynamical lift force $L(t)$ and aerodynamical torsional moment $M(t)$ are involved, which satisfy

$$
L=-l \int_{\Gamma_{W t}} \sigma_{2 j} n_{j} d S, \quad M=l \int_{\Gamma_{W t}} \sigma_{i j} n_{j} r_{i}^{\text {ort }} d S,
$$

where $\sigma_{i j}=\rho\left[-p \delta_{i j}+2 \nu S_{i j}\right], r_{1}^{\text {ort }}=-\left(x_{2}-x_{2}^{\mathrm{EA}}\right), r_{2}^{\text {ort }}=x_{1}-x_{1}^{\mathrm{EA}}, l$ denotes the considered depth of the aerofoil section, and $x^{\mathrm{EA}} e=\left(x_{1}^{\mathrm{EA}}, x_{2}^{\mathrm{EA}}\right)$ is the position of EA of the aerofoil at the time instant $t$, see Figure 1

\section{NUMERICAL APPROXIMATION}

In this section, the approximation of the considered mathematical model of transitional turbulent flow is shown. The time discretization is based on the backward difference formula and for the spatial discretization the stabilized FEM is used. Further, the four equations of the transitional model are discretized in time, linearized and stabilized using the streamline upwind/Petrov Galerkin (SUPG) method. Let us moreover mention that in the computations for the $k-\omega$ equations the (nonlinear) crosswind diffusion is also applied in order to suppress non-physical undershoots/overshoots, see [19]. 
3.1. Time discretization. We consider the equidistant partition $t_{j}=j \Delta t$ of the time interval $I$ with a time step $\Delta t>0$, and denote the approximations $\boldsymbol{u}^{j} \approx \boldsymbol{u}\left(\cdot, t_{j}\right)$, $p^{j} \approx p\left(\cdot, t_{j}\right)$ and similarly $k^{j}=k\left(\cdot, t_{j}\right), \omega^{j}=\omega\left(\cdot, t_{j}\right), \gamma^{j}=\gamma\left(\cdot, t_{j}\right), \overline{R e}_{\theta t}{ }^{j}=\overline{R e}_{\theta t}\left(\cdot, t_{j}\right)$. Moreover, we approximate the domain velocity $\boldsymbol{w}_{D}$ at time level $t_{j}$ by $\boldsymbol{w}_{D}^{j}$. We shall focus on the description of the discretization at a time instant $t_{n+1}$, which is kept fixed throughout this section. For the sake of simplicity the subscript $t_{n+1}$ shall be omitted, i.e. $\Omega=\Omega_{t_{n+1}}$. Further, we shall denote by $Q=L^{2}(\Omega)$ the Lebesgue space, by $\boldsymbol{W}=\boldsymbol{H}^{1}(\Omega)$ the Sobolev space and by $\boldsymbol{X}$ the space of test functions defined by $\boldsymbol{X}=\left\{\boldsymbol{z} \in \boldsymbol{W}: \boldsymbol{z}=0\right.$ on $\left.\Gamma_{D} \cup \Gamma_{W t_{n+1}}\right\}$. Then the time derivatives in equations

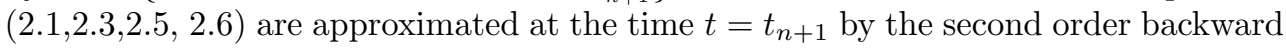
difference formula, i.e.

$$
\left.\frac{\partial \varphi}{\partial t}\right|_{t_{n+1}} \approx \frac{3 \varphi^{n+1}-4 \varphi^{n}+\varphi^{n-1}}{2 \Delta t} .
$$

Here, let us mention that for ALE approach the ALE derivative needs to be approximated at this place.

3.2. Spatial discretization of flow model. The weak formulation of the time discretized Reynolds averaged Navier-Stokes equations is obtained by the multiplication of the equations (2.1) by a test function $\boldsymbol{z} \in \mathcal{X}$, integration over the domain $\Omega_{t}$, application of the Green's theorem and using approximation (3.1). The (spatial) weak formulation reads: Find $U^{n+1}=\left(\boldsymbol{u}^{n+1}, p^{n+1}\right) \in \boldsymbol{W} \times Q$ such that $\boldsymbol{u}$ approximately satisfies the boundary conditions $(2.2 \mathrm{a}, \mathrm{b})$ and

$$
a\left(U^{n+1}, V\right)=L(V) .
$$

holds for all $V=(\boldsymbol{z}, q) \in \mathcal{X} \times Q$. The non-linear form $a(\cdot, \cdot)$ and the linear form $L(\cdot)$ are defined for any $U=(\boldsymbol{u}, p) \in \boldsymbol{W} \times Q$ and $V=(\boldsymbol{z}, q) \in \mathcal{X} \times Q$,

$$
\begin{aligned}
a(U, V) & =\left(\frac{3 \boldsymbol{u}}{2 \Delta t}+(\overline{\boldsymbol{w}} \cdot \nabla) \boldsymbol{u}, \boldsymbol{z}\right)_{\Omega}+\left(\nu_{\mathrm{eff}} \boldsymbol{S}(\boldsymbol{u}), \boldsymbol{S}(\boldsymbol{z})\right)_{\Omega}+(\nabla \cdot \boldsymbol{u}, q)_{\Omega}-(p, \nabla \cdot \boldsymbol{z})_{\Omega}, \\
L(V) & =\frac{4}{2 \Delta t}\left(\hat{\boldsymbol{u}}^{n}, \boldsymbol{z}\right)_{\Omega}-\frac{1}{2 \Delta t}\left(\hat{\boldsymbol{u}}^{n-1}, \boldsymbol{z}\right)_{\Omega},
\end{aligned}
$$

where $\tilde{\boldsymbol{u}}^{k}=\boldsymbol{u}^{k} \circ \mathcal{A}_{t_{k}} \circ \mathcal{A}_{t_{n+1}}^{-1}$. In order to approximate the problem 3.2 , the spaces $\boldsymbol{X}, \boldsymbol{W}$ and $Q$ are approximated by finite element subspaces $\boldsymbol{X}_{\Delta}, \boldsymbol{W}_{\Delta}$ and $Q_{\Delta}$, respectively.

Here, the Taylor-Hood family of finite elements are used, defined over an admissible triangulation $\mathcal{T}_{\Delta}$ of the computational domain $\Omega=\Omega_{t_{n+1}}$. In order to stabilize the method the fully stabilized scheme (see [16]) is used, which consists of SUPG and pressure stabilizing/Petrov Galerkin (PSPG) stabilization combined with the div-div stabilization, see [16].

The stabilized discrete problem reads: Find $U=\left(\boldsymbol{u}_{\Delta}^{n+1}, p_{\Delta}^{n+1}\right) \in \boldsymbol{W}_{\Delta} \times Q_{\Delta}$ such that $\boldsymbol{u}^{n+1}$ satisfies approximately the Dirichlet boundary conditions $\left.2.2, \mathrm{a}, \mathrm{b}\right)$ and

$$
a(U ; U, V)+\mathcal{L}(U, V)+\mathcal{P}(U, V)=L(V)+\mathcal{F}(V),
$$


holds for all $V=(\boldsymbol{z}, q) \in \boldsymbol{X}_{\Delta} \times Q_{\Delta}$, where the terms $\mathcal{L}$ and $\mathcal{F}$ are the SUPG/PSPG terms defined by

$$
\begin{array}{r}
\mathcal{L}(U, V)=\sum_{K \in \mathcal{T}_{\Delta}} \delta_{K}\left(\frac{3 \boldsymbol{u}}{2 \Delta t}-\nu \Delta \boldsymbol{u}+(\boldsymbol{v} \cdot \nabla) \boldsymbol{u}+\nabla p, \Psi\right)_{K}, \\
\mathcal{F}(V)=\sum_{K \in \mathcal{T}_{\Delta}} \delta_{K}\left(\frac{1}{2 \Delta t}\left(4 \tilde{\boldsymbol{u}}^{n}-\tilde{\boldsymbol{u}}^{n-1}\right), \Psi\right)_{K},
\end{array}
$$

where the function $\overline{\boldsymbol{w}}^{n+1}=\boldsymbol{u}^{*}-\boldsymbol{w}_{D}^{n+1}$ stands for the transport velocity, and $\Psi=$ $\left(\overline{\boldsymbol{w}}^{n+1} \cdot \nabla\right) \boldsymbol{z}+\nabla q$. The div-div stabilizing terms $\mathcal{P}(U, V)$ read

$$
\mathcal{P}(U, V)=\sum_{K \in \mathcal{T}_{\Delta}} \tau_{K}(\nabla \cdot \boldsymbol{u}, \nabla \cdot \boldsymbol{z})_{K} .
$$

Here, the following choice of the stabilizing parameters $\tau_{K}$ and $\delta_{K}$ based on the local element length $h_{K}$ is used for the Taylor-Hood family of finite elements, $\tau_{K}=$ $\max _{x \in \Omega}\|\boldsymbol{u}(x)\|_{2}, \delta_{K}=h_{K}^{2} / \tau_{K}$.

3.3. Spatial discretization of the turbulence/transitional model. Furthermore, the complete transitional model consisting of equations [2.3, 2.5], 2.6 is step-by-step time discretized, weakly formulated, and stabilized formulation is introduced. The time derivatives in equations $2.3, \sqrt{2.5}$ and $(2.6)$ are approximated using the 2nd order backward difference formula (3.1), the equations for $k, \omega, \gamma$ and $\overline{R e}_{\theta t}$ are multiplicated by $\varphi_{k}, \varphi_{\omega}, \varphi_{\gamma}$ and $\varphi_{\overline{R e}}$, respectively, integrated over $\Omega_{t}$, and Green's theorem is applied. The function spaces are then approximated by their finite element counterparts consisting of piecewise linear functions defined over the triangulation $\mathcal{T}_{\Delta}$. The stabilized formulation is obtained using a linearization of the non-linear terms and an application of the SUPG stabilization procedure.

\section{Numerical Results}

The described method was verified using several test cases of laminar, turbulent and transitional flow, see [9], 19], 22. Here, the method was applied to two test cases of aeroelastic simulation of flow induced aerofoil vibrations and the transient response to the sudden change of flow conditions (gust).

4.1. Aeroelastic simulations for NACA 0012. First, the numerical simulation of the fully coupled aeroelastic problem of flow induced vibrations of the aerofoil NACA 0012 was addressed. The following parameters of the flowing air and the profile were used: $m=8.66 \times 10^{-2} \mathrm{~kg}, S_{\varphi}=-7.797 \times 10^{-4} \mathrm{kgm}, I_{\varphi}=4.87 \times 10^{-4} \mathrm{~kg} \mathrm{~m}^{2}$, $k_{h}=105.1 \mathrm{~N} / \mathrm{m}, k_{\varphi}=3.696 \mathrm{Nm} / \mathrm{rad}, d=0.05 \mathrm{~m}, c=0.3 \mathrm{~m}, \rho=1.225 \mathrm{~kg} / \mathrm{m}^{3}$, $\nu=1.5 \times 10^{-5} \mathrm{~m} / \mathrm{s}^{2}$, see also [9]. The elastic axis EA is located at $40 \%$ of the profile. The numerical simulation was performed for the sub- and also close to critical far field velocity $U_{\infty}=37.7 \mathrm{~m} \mathrm{~s}^{-1}$ determined by a linear approach.

The verification of the applied numerical method was done with the main attention paid to the resulting aeroelastic response. Figure 2 shows the aeroelastic response of 

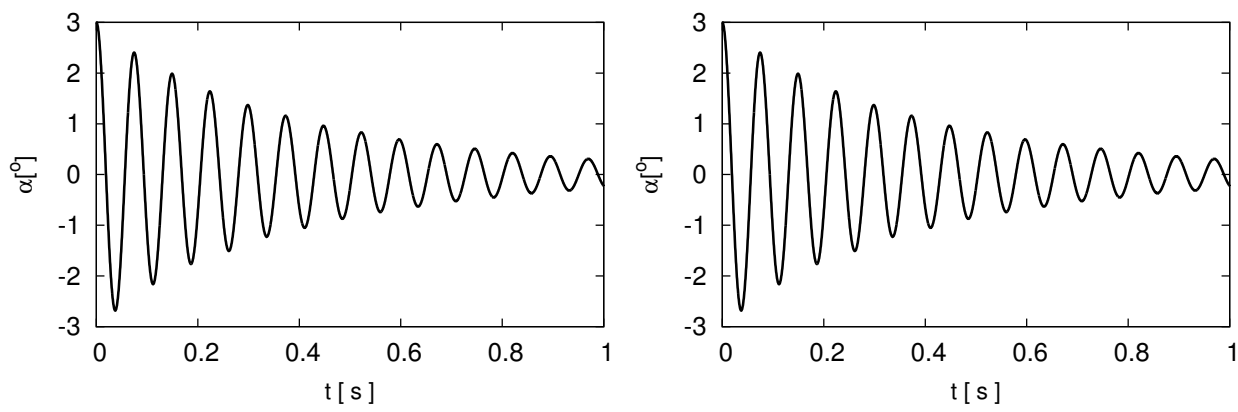

Figure 2. Aeroelastic response of NACA 0012 aerofoil for sub-critical far field velocity $U_{\infty}=10 \mathrm{~m} / \mathrm{s}$
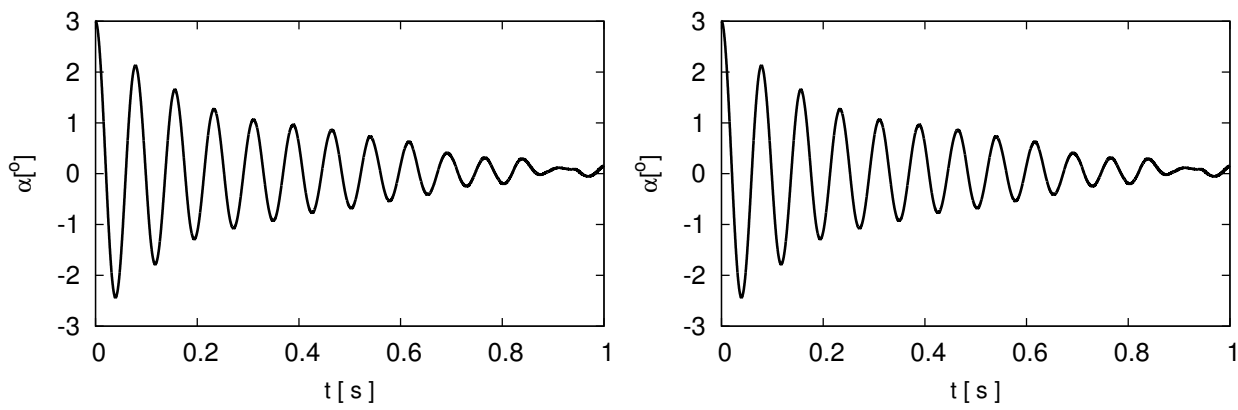

Figure 3. Aeroelastic response of NACA 0012 aerofoil for sub-critical far field velocity $U_{\infty}=20 \mathrm{~m} / \mathrm{s}$

the fully coupled FSI problem for far field velocity $U_{\infty}=10 \mathrm{~m} / \mathrm{s}$. The aeroelastic response predict stronger aerodynamical damping for flow velocity $U_{\infty}=20 \mathrm{~m} / \mathrm{s}$, see Figure 3. For higher flow velocities closer to the critical velocity the aeroelastic response seems to predict again decrease of the aerodynamical damping for $U_{\infty}=$ $35 \mathrm{~m} / \mathrm{s}$, see Figure 4. For $U_{\infty}=38 \mathrm{~m} / \mathrm{s}$ the aeroelastic system is only weakly damped, see Figure 5, which agrees well with the results in the literature.

4.2. Gust response. The developed numerical method was applied on numerical approximation of flow around a flexibly supported aerofoil subject to the change of flow conditions, see [21]. The results are compared with the previous study of the authors [20, where turbulent and non-turbulent flows were considered without modelling of the turbulent-laminar flow transition. The considered aerofoil shape is given by

$$
z=\frac{k_{\mathrm{KT}}\left(c_{\mathrm{KT}}-1\right)}{\left((Z-1) /\left(Z-c_{\mathrm{KT}}\right)\right)^{k_{\mathrm{KT}}-1}}, \quad k_{\mathrm{KT}}=\frac{360-\alpha_{T}}{180}
$$

where $Z=X+i Y$ and $z=x+i y$ are complex variables describing the unit circle and the aerofoil shape in $X-Y$ and $x-y$ complex planes, respectively. The constants $c_{\mathrm{KT}}=-0.89-0.11 i$ and $\alpha_{T}=2 \mathrm{deg}$ determine the aerofoil shape (A1), see Figure 1. The following values were used $m=2 \times 10^{-4} \mathrm{~kg}, I_{\alpha}=1.2 \times 10^{-7} \mathrm{~kg} \mathrm{~m} \mathrm{~m}^{2}$ and $S_{\alpha}=$ 

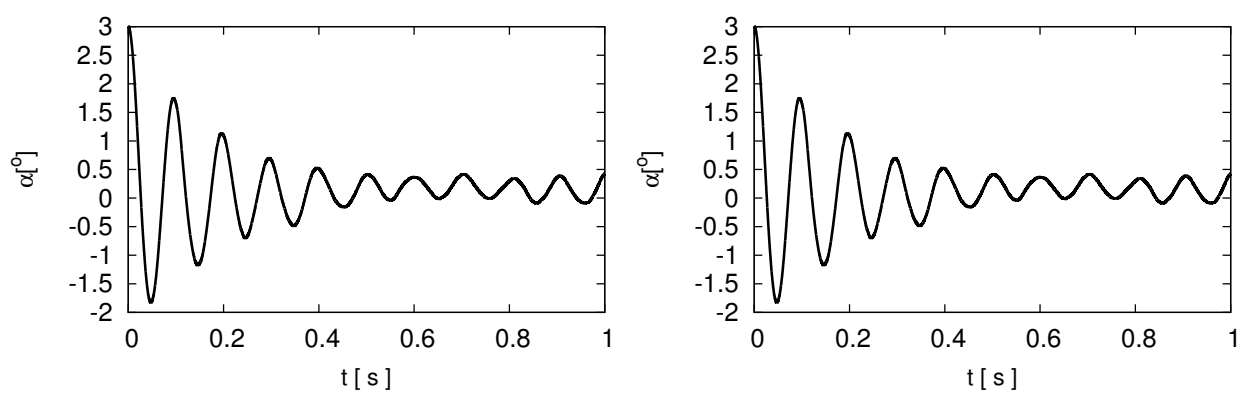

Figure 4. Aeroelastic response of NACA 0012 aerofoil for sub-critical far field velocity $U_{\infty}=35 \mathrm{~m} / \mathrm{s}$
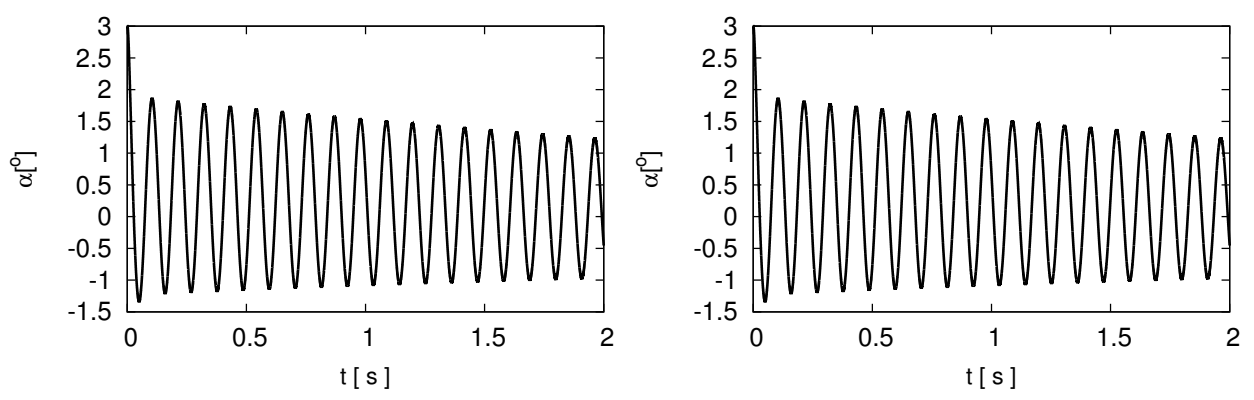

Figure 5. Aeroelastic response of NACA 0012 aerofoil for sub-critical far field velocity $U_{\infty}=38 \mathrm{~m} / \mathrm{s}$

$2 \times 10^{-6} \mathrm{~kg} \mathrm{~m}$. The aerofoil chord was $c=0.1 \mathrm{~m}$, the elastic axis was located at $30 \%$ of the chord, and the depth of the aerofoil section was $l=0.03 \mathrm{~m}$. The stiffness coefficients of the springs were $k_{h}=26 \mathrm{~N} / \mathrm{m}, k_{\alpha}=0.29 \mathrm{~N} \mathrm{~m} / \mathrm{rad}$.

The air density was $\rho=1.225 \mathrm{~kg} \mathrm{~m}^{-3}$ and the air kinematic viscosity was $\nu=$ $1.453 \times 10^{-5} \mathrm{~m}^{2} / \mathrm{s}$. The inlet turbulence intensity was $1 \%\left(k=1.5 \times 10^{-4} U_{\infty}^{2}, \omega=\right.$ $10 \mathrm{~s}^{-1}$ on $\left.\Gamma_{I}\right)$. The finite element triangular mesh was used, anisotropically refined nearby the boundary in order to well capture the turbulent boundary layer, wake and also the separation region. The described stabilized FEM was used, and the computations were performed on the coarse and the fine mesh. A vertical gust of $1 \mathrm{~s}$ duration was considered as a sudden perturbation of the inlet velocity $U_{\infty}=15 \mathrm{~m} / \mathrm{s}$, i.e.

$$
V_{g}(t)=\frac{V_{G}}{2}\left(1+\cos \left(\pi\left(t-t_{0}\right)\right)\right)
$$

for $t \in\left[t_{0}, t_{0}+1\right]$ and $V_{g}(t) \equiv 0$ otherwise. Here, $V_{G}=1.5 \mathrm{~m} \mathrm{~s}^{-1}$ and $V_{G}=5 \mathrm{~m} \mathrm{~s}^{-1}$ were considered for the light and heavy gusts, respectively. The aeroelastic aerofoil responses $h(t)$ and $\alpha(t)$ numerically simulated in the time domain for the light gust are shown in Figure 6 . The numerically simulated aerofoil responses $h(t)$ and $\alpha(t)$ for the heavy gust are shown in Figure 7. The results for the transitional flow model are close to the simulations using the turbulence model, but before the gust starts and 

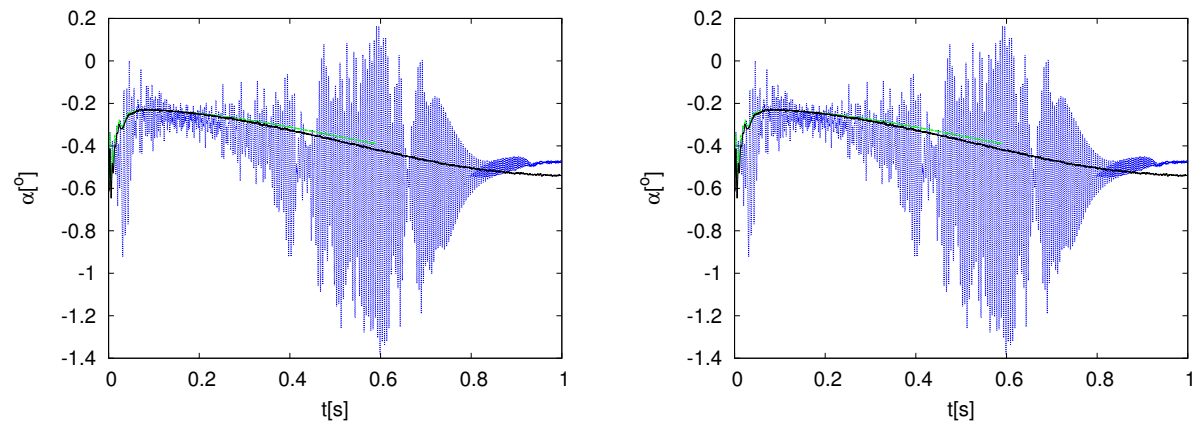

Figure 6. Aeroelastic response to the light gust: Comparison of results computed by laminar, turbulent and transitional models
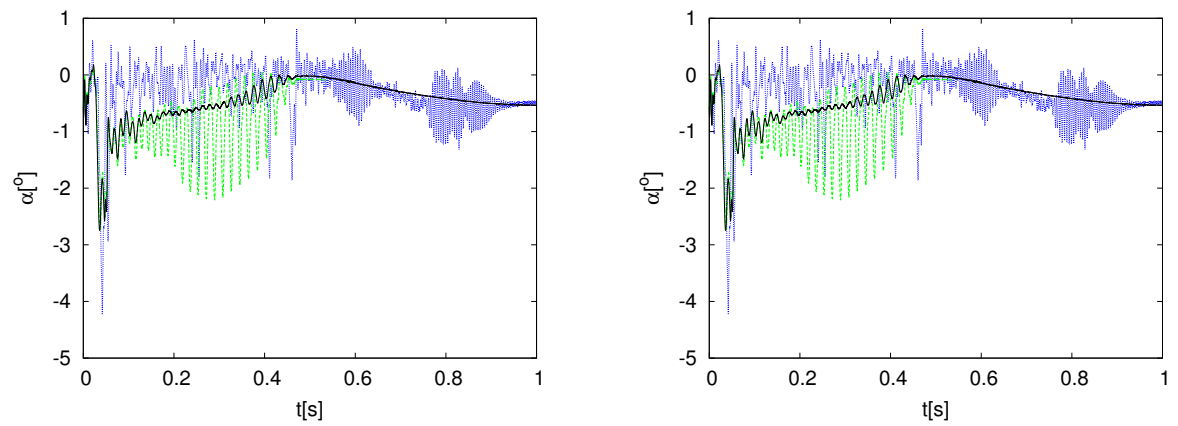

Figure 7. Aeroelastic response to the heavy gust: Comparison of results computed by laminar, turbulent and transitional models
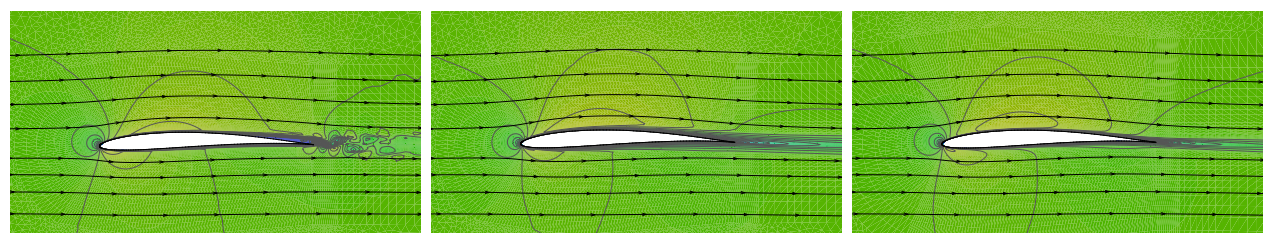

Figure 8. Comparison of velocity flow patterns for the laminar (left), turbulent (middle) and transitional(right) models just before the gust starts
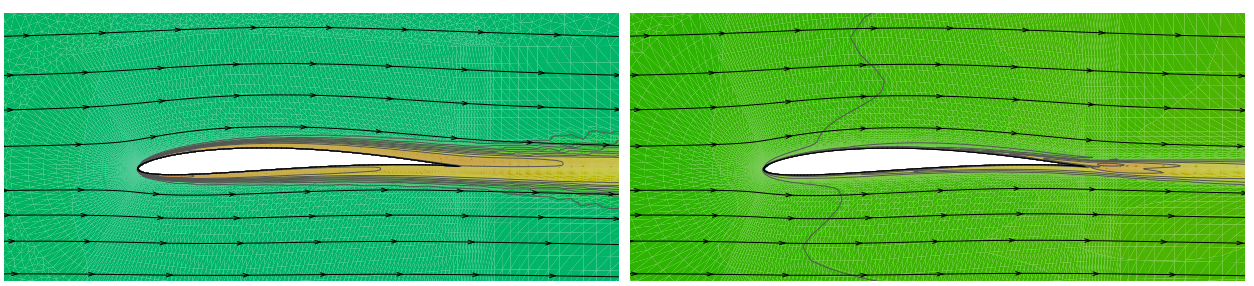

Figure 9. Comparison of the turbulent kinetic energy $k$ distribution for the turbulent (left) and transitional (right) models just before the gust starts 
also when it disappears, the fluid flow becomes almost laminar on the aerofoil surface. The comparison of the flow velocity patterns just before the gust starts computed by the laminar, turbulent and transitional model are shown in Figure 8 See also turbulent kinetic energy pattern at the same time instant shown in Figure 9, where the most of the turbulent kinetic energy for the transitional model is in a far wake and almost zero turbulent kinetic energy is at the aerofoil surface. On the other hand
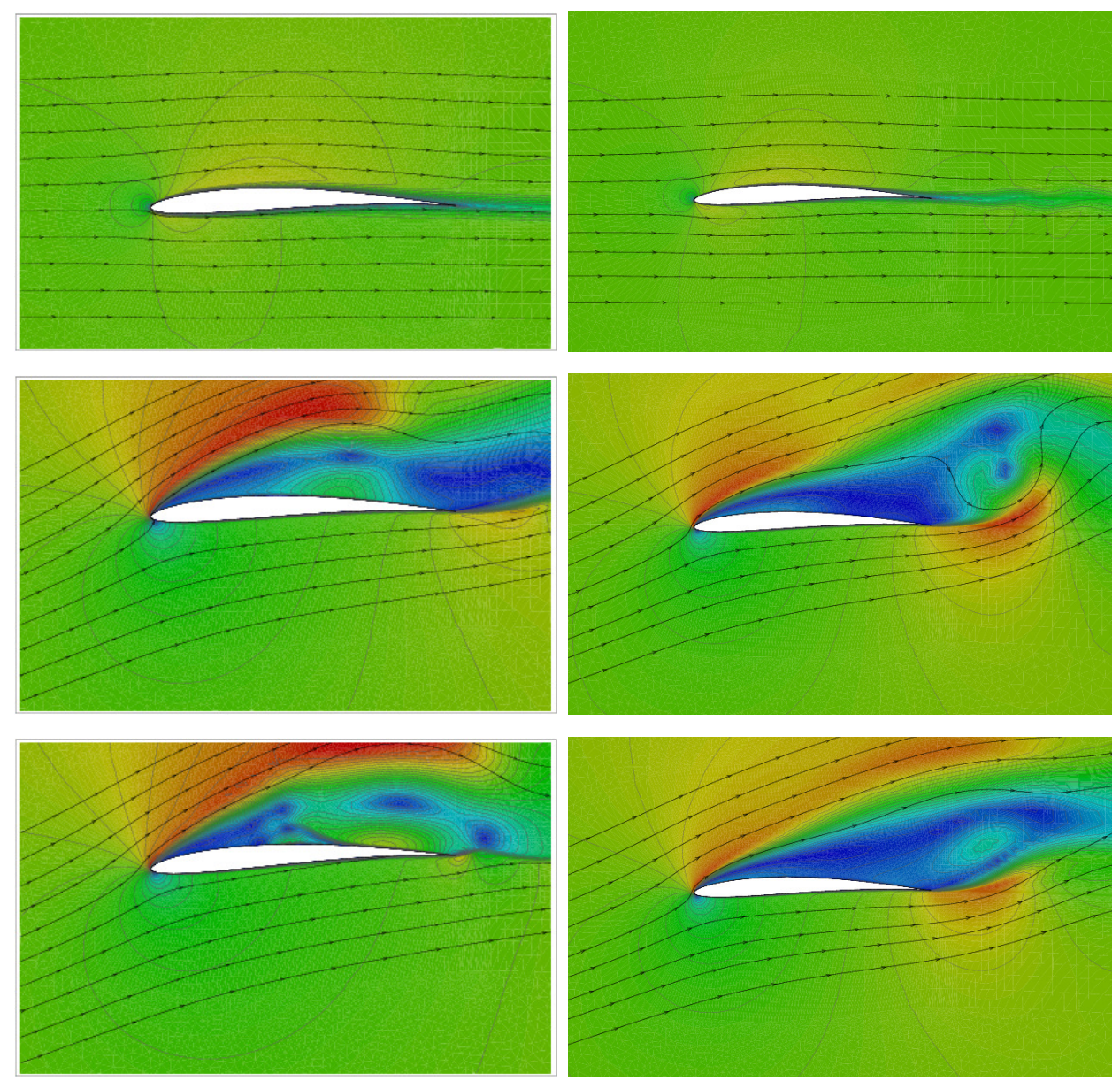

Figure 10. Heavy gust aeroelastic response: comparison of flow velocity patterns for turbulent (left) and transitional (right) models at three time instants

the flow velocity patterns shown in Figure 10 indicate that the transitional model is much closer to the turbulent model for high displacement of the aerofoil. 


\section{Conclusion}

A new original FEM taking into account transition of the laminar to turbulent flow was developed and successfully applied on the FSI problem of flow induced vibrations for a 2-DOF aerofoil. The results were computed by the developed in-house software and the fully coupled FSI problem was solved. In addition to implementation of the laminar-turbulent flow transition, the developed method was also modified for application in the case of case of time dependent computational domain using the ALE method. First, the aeroelastic simulation were performed for the NACA 0012 aerofoil, whose vibrations were induced by the prescribed initial conditions. The results of the transitional model agree well with the reference results [9]. Further, the simulation of an 2-DOF aerofoil loaded by a sudden gust was performed. The results of the described method was compared with reference results 21]. The transitional model was shown to "switch" between the laminar/turbulent model in dependence on the displacements, i.e. the results of the transitional model correspond better to the results of the laminar model for the case of small displacements (light gust), whereas for large displacements (heavy gust) the results agree better with the results obtained by the fully turbulent model. This influence is particularly important for small displacements (light gust case).

Acknowledgment. This work was supported by project No. P101/12/1271 of the Czech Science Foundation.

\section{REFERENCES}

1. Dowell, E. H. and Clark, R. N.: A Modern Course in Aeroelasticity. Solid mechanics and its applications, Kluwer Academic Publishers, Dordrecht, Boston, 2004.

2. Verhoosel, C., Scholcz, T., and Hulshoff, S.: Uncertainty and reliability analysis of fluid-structure stability boundaries. AIAA Journal, 47, (2009), 91-104.

3. Jones, D. and Gaitonde, A.: Future fast methods for loads calculations: The 'FFAST' project. In D. Knorzer and J. Szodruch (eds.), Innovation for Sustainable Aviation in a Global Environment, pp. 110-115, IOS Press BV, 2012.

4. Berci, M., Gaskell, P. H., Hewson, R., and Toropov, V.: A semi-analytical model for the combined aeroelastic behaviour and gust response of a flexible aerofoil. Journal of Fluids and Structures, 38, (2013), 3-21.

5. Sucipto, T., Berci, M., and Krier, J.: Gust response of a flexible typical section via high- and (tuned) low-fidelity simulations. Computers 85 Structures, 122, (2013), 202-216.

6. Vos, J., Rizzi, A., DarracQ, D., and Hirschel, E.: Navier-Stokes solvers in European aircraft design. Progress in Aerospace Sciences, 38(8), (2002), 601-697.

7. Buka ̌, M., Čanič, S., and Muna, B.: A partitioned scheme for fluid-composite structure interaction problems. Journal of Computational Physics, 281, (2015), 493-517.

8. Nomura, T. and Hughes, T. J. R.: An arbitrary Lagrangian-Eulerian finite element method for interaction of fluid and a rigid body. Computer Methods in Applied Mechanics and Engineering, 95, (1992), 115-138. 
9. Sváček, P., Feistauer, M., and Horáček, J.: Numerical simulation of flow induced airfoil vibrations with large amplitudes. Journal of Fluids and Structures, 23(3), (2007), 391-411.

10. Boffi, D. and Gastaldi, L.: Stability and geometric conservation laws for ALE formulations. Computational Methods in Applied Mechanical Engineering, 193, (2004), 47174739 .

11. Farhat, C. and Geuzaine, P.: Design and analysis of robust ALE time-integrators for the solution of unsteady flow problems on moving grids. Computational Methods in Applied Mechanical Engineering, 193, (2004), 4073-4095.

12. Gresho, P. M. and Sani, R. L.: Incompressible Flow and the Finite Element Method. Wiley, Chichester, 2000.

13. Roos, H.-G., Stynes, M., and Tobiska, L.: Robust Numerical Methods for Singulary Perturbed Differential Equations: Convection Diffusion and Flow Problems, vol. 24. Springer, Berlin, Heidelberg, 2008.

14. Lube, G. and RAPIN, G.: Residual-based stabilized higher-order FEM for advectiondominated problems. Computer Methods in Applied Mechanics and Engineering, 195(3336), (2006), 4124-4138.

15. Linke, A., Rebholz, L. G., and Wilson, N. E.: On the convergence rate of graddiv stabilized Taylor-Hood to Scott-Vogelius solutions for incompressible flow problems. Journal of Mathematical Analysis and Applications, 381(2), (2011), 612-626.

16. Gelhard, T., Lube, G., Olshanskit, M. A., and Starcke, J.-H.: Stabilized finite element schemes with LBB-stable elements for incompressible flows. Journal of Computational and Applied Mathematics, 177, (2005), 243-267.

17. Feistauer, M., Horáček, J., RŮŽı̌̌Ka, M., and SváČek, P.: Numerical analysis of flow-induced nonlinear vibrations of an airfoil with three degrees of freedom. Computers \& Fluids, 49(1), (2011), 110 - 127.

18. Wilcox, D. C.: Turbulence Modeling for CFD. DCW Industries, 1993.

19. Feistauer, M., Horáček, J., and Sváček, P.: Numerical simulation of vibrations of an airfoil induced by turbulent flow. Communications in Computational Physics, 17(1), (2015), 146-188.

20. SvÁČEK, P. and HorÁČEK, J.: On mathematical modeling of fluid-structure interactions with nonlinear effects: Finite element approximations of gust response. Journal of Computational and Applied Mathematics, 273(0), (2015), 394 - 403.

21. Berci, M., Mascetti, S., Incognito, A., Gaskell, P. H., and Toropov, V. V.: Gust response of a typical section via CFD and analytical solutions. In J. C. F. Pereira and A. Sequeira (eds.), ECCOMAS CFD 2010, V. European Conference on Computational Fluid Dynamics, 2010, p. 10 pp.

22. HoráČEK, J. and SvÁČEK, P.: Finite element simulation of a gust response of an ultralight 2-DOF airfoil. In Proceedings of the ASME 2014 Pressure Vessels 86 Piping Conference, PVP2014-28390, Anaheim, California, USA, 2014, 10 pp.

23. Walters, D. K. and Cokluat, D.: A three-equation eddy-viscosity model for Reynoldsaveraged Navier-Stokes simulations of transitional flow. Journal of Fluids Engineering, 130(12), (2008), 121401. 
24. Suluksna, K. and Juntasaro, E.: Assessment of intermittency transport equations for modeling transition in boundary layers subjected to freestream turbulence. International Journal of Heat and Fluid Flow, 29(1), (2008), 48 - 61.

25. Suzen, Y., Huang, P., Hultgren, L., and Ashpis, D.: Predictions of separated and transitional boundary layers under low-pressure turbine airfoil conditions using an intermittency transport equation. Journal of Turbomachinary-Transactions of the ASME, 125(3), (2003), 455-464.

26. Menter, F., Langtry, R., and Völker, S.: Transition modelling for general purpose CFD codes. Flow, Turbulence and Combustion, 77(1-4), (2006), 277-303.

27. Suzen, Y., Huang, P., Hultgren, L. S., and Ashpis, D. E.: Predictions of separated and transitional boundary layers under low-pressure turbine airfoil conditions using an intermittency transport equation. Journal of Turbomachinery, 125(3), (2003), 455-464.

28. Kim, S. W., Zaman, K. B. M. Q., and PAndA, J.: Numerical investigation of unsteady transitional flow over oscillating airfoil. Journal of Fluids Engineering-Transactions of the ASME, 117(1), (1995), 10-16.

29. Weber, S., Jones, K., Ekaterinaris, J., and Platzer, M.: Transonic flutter computations for the NLR 7301 supercritical airfoil. Aerospace Science and Technology, 5, (2001), 293-304.

30. Poirel, D., MÉtivier, V., and Dumas, G.: Computational aeroelastic simulations of self-sustained pitch oscillations of a NACA0012 at transitional Reynolds numbers. Journal of Fluids and Structures, 27, (2011), 1262-1277.

31. Yuan, W., Poirel, D., Wang, B., and Benaissa, A.: Effect of freestream turbulence on airfoil limit-cycle oscillations at transitional Reynolds numbers. Journal of Aircraft, 52(4), (2015), 1214-1225.

32. Pope, S. B.: Turbulent Flows. Cambridge University Press, Cambridge, 2000.

33. Menter, F. R.: Two-equations eddy-viscosity turbulence models for engineering applications. AIAA Journal, 32(8), (1994), 1598-1605.

34. KoK, J. C.: Resolving the dependence on free-stream values for the k-omega turbulence model. Tech. rep., National Aerospace Laboratory NLR, 1999.

35. Langtry, R. B. and Menter, F. R.: Correlation-based transition modeling for unstructured parallelized computational fluid dynamics codes. AIAA Journal, 47(12), (2009), 2894-2906. 